\title{
UNIFORMIDADE DE DISTRIBUIÇÃO DE ÁGUA NA SUPERFÍCIE E NO PERFIL DO SOLO IRRIGADO COM O MICROASPERSOR RAIN-BIRD QN-14 ${ }^{1}$
}

\author{
Tarcísio Nascimento ${ }^{2}$, José Monteiro Soares $^{2}$ \& Carlos Alberto Vieira de Azevedo 3
}

\begin{abstract}
RESUMO
Avaliando-se, o Coeficiente de Uniformidade de Christiansen (CUC) das lâminas de água aplicadas na superfície e de suas respectivas distribuições no perfil do solo, nas profundidades de 0 - 0,20, 0,20 - 0,40 e 0,40 - 0,60 m, para o microaspersor Rain-Bird QN-14, em condições de campo, constatou-se que os valores de CUC foram da ordem de 42,61, 64,90, 79,04 e 84,06\%, respectivamente. Observou-se também, que os valores de CUC das lâminas de água redistribuídas no perfil do solo aumentou com a profundidade. O baixo valor de CUC para precipitação das lâminas de água na superfície do solo não exerceu influência nos coeficientes de uniformidade de distribuição da umidade no perfil do solo, exceto para a camada de $0-0,20 \mathrm{~m}$.
\end{abstract}

Palavras-chave: irrigação, microaspersão, coeficiente de uniformidade, lâminas de irrigação

\section{DISTRIBUTION UNIFORMITY OF WATER AT THE SURFACE AND IN THE SOIL PROFILE IRRIGATED WITH MICROSPRINKLER RAIN-BIRD QN-14}

\begin{abstract}
In this study the Christiansen Uniformity Coefficient (CUC), for the water application at the surface and in the soil profile at the depths of $0-0.20,0.20-0.40$ and $0.40-0.60 \mathrm{~m}$, for microsprinkler Rain-bird QN-14 operating under field conditions was evaluated. The values of CUC at the respective depths were $42.61,64.90,79.04$ and $84.06 \%$ and at the surface an average coefficient of $42.61 \%$ was obtained. It may be noted that the uniformity in the soil profile increases with the depth due to redistribution of water. The low value of uniformity of precipitations at the soil surface did not influence the uniformity distribution of the moisture in the soil profile, except for $0-0.20 \mathrm{~m}$ depth.
\end{abstract}

Key words: irrigation, microsprinkle, uniformity coefficient, water depths

\section{INTRODUÇÃO}

A irrigação tem sido uma das práticas mais utilizadas na agricultura moderna com o objetivo de aumentar a produção agrícola, porém sua maximização em relação à água aplicada é função da concepção e dimensionamento adequados do sistema de irrigação e da sua operação que propicie uma uniformidade de aplicação d'água adequada.

Conforme Clemmens (1991) apud Lucena (1993), os sistemas de irrigação são, por natureza, desuniformes em sua aplicação de água. Para superar este problema, dependendo da disponibilidade de água na região, pode-se aplicar uma maior

\footnotetext{
${ }^{1}$ Parte da dissertação de Mestrado apresentada pelo primeiro autor à UFPB

${ }^{2}$ M.Sc. em Irrigação e Drenagem, Embrapa Semi-Árido, Petrolina, PE, Fone: (081) 8621711 - Ramal: 193, Fax: (081) 862 1744, E-mail: tarcizio@cpatsa.embrapa.br,monteiro@cpatsa.embrapa.br

${ }^{3}$ Ph.D. em Irrigação e Drenagem, DEAg/CCT/UFPB, Av. Aprigio Veloso 882, Bodocongó, CEP 58109 - 970, Campina Grande, PB, Fone: (083) 310 1318, Fax: (083) 310 1185, E-mail: cazevedo@ deag.ufpb.br
} 
lâmina de água para assegurar a lâmina requerida pela cultura ao longo do seu ciclo fenológico. Entretanto, quando os recursos hídricos são escassos, uma alternativa seria estabelecer uma irrigação com uma desuniformidade de aplicação aceitável na zona radicular, desde que não se comprometa substancialmente a produtividade da cultura considerada.

Um dos principais parâmetros em avaliações do desempenho de um sistema de irrigação por aspersão e microaspersão é a uniformidade de distribuição de água; no entanto, na maioria das vezes essas avaliações tem-se preocupado em verificar a uniformidade de aplicação d'água pelo sistema apenas na superfície do solo, assumindo-se que esta representa a uniformidade de distribuição da água infiltrada, o que implica em levar em conta apenas o fluxo unidimensional descendente, ignorando, assim, toda a complexidade do movimento d'água no perfil do solo (Goldhamer et al., 1985). Segundo Reichardt (1985), a tendência expontânea e universal de toda matéria na natureza, é assumir um estado de energia mínima, e a água obedecendo a esse conceito, move-se constantemente no sentido da diminuição de seu potencial, promovendo, então, uma redistribuição de umidade no perfil do solo.

Segundo Choudhury \& Millar (1983), a caracterização da retenção e das propriedades transmissoras da água no solo, é um requisito necessário para a descrição qualitativa do perfil do solo, com relação ao balanço hídrico. Esses autores mencionam ainda que quando o solo é manejado sob condições de irrigação, os reflexos dos processos físicos, químicos e biológicos são de grande importância agrícola. Hills (1982) apud Silva (1988) afirma que o solo controla o movimento da água especialmente através de seus potenciais de pressão e mátrico; Pereira et al. (1974) afirmam que a camada superficial do solo é de grande importância no balanço hídrico e esta interage de forma significativa nos movimentos da água tanto superficial como subterrânea.

Firake \& Salunkha (1992), avaliando a influência da pressão de serviço $(150,200$ e $250 \mathrm{kPa})$ de um sistema de irrigação por microaspersão, em condições de campo, na distribuição da umidade no solo, observaram um decréscimo na umidade com um aumento da pressão ao longo do perfil do solo, mostrando que quanto maior a pressão de serviço do microaspersor maior é o tempo de operação do sistema requerido para saturar a zona radicular. Ramos et al. (1993), avaliando também os efeitos de quatro vazões e quatro volumes de água aplicados pontualmente num Latossolo Vermelho Álico, observaram que os maiores volumes de água aplicados alcançaram maiores profundidades nos perfis de infiltração para uma mesma vazão, enquanto a maior vazão para um mesmo volume aplicado promoveu um maior avanço horizontal.

Medeiros (1987), estudando a aplicação de três volumes de água para elevar a umidade do solo à capacidade de campo nas profundidades de 25,50 e $75 \mathrm{~cm}$, obteve áreas molhadas de $11,89,12,79$ e $13,43 \mathrm{~m}^{2}$, respectivamente. Constatou ainda, que após as irrigações a frente de umedecimento nas respectivas camadas, alcançou as profundidades médias de 31,40 e $48 \mathrm{~cm}$, tendo a eficiência de armazenamento sido da ordem de $66,51 \mathrm{e}$ $41 \%$, respectivamente, enquanto a eficiência de aplicação média foi de $80,27 \%$.
Na microaspersão, vários pesquisadores têm simulado ou encontrado em campo baixos valores para o Coeficiente de Uniformidade de Christiansen (CUC), na superfície do solo. Souza et al. (1993) avaliando um sistema de irrigação por microaspersão encontraram valores para CUC, na superfície do solo, que variaram entre 25,5 a $36,8 \%$. Através de simulações, Rocha \& Lima (1996) obtiveram para o microaspersor Antelco, modelo RRN30, valores para CUC variando entre 15 a $60 \%$. Em testes de campo, Lima \& Alves (1994) utilizando também microdifusores e microaspersores numa malha de 11 x $11 \mathrm{~m}$, obtiveram valores de CUC inferiores a $25 \%$ para ambos os emissores na superfície do solo, enquanto que para o perfil do solo, os valores destes coeficientes elevaram-se para uma faixa entre 40 a $50 \%$ para microdifusores e para um intervalo de 52 a $57 \%$ para microaspersores. Esses resultados mostram que a uniformidade de precipitação na superfície do solo, não é um parâmetro adequado para efeito de dimensionamento e manejo deste sistema de irrigação, principalmente em se tratando de irrigação de fruteiras, em que o uso de microaspersores isolados, não proporciona a superposição de precipitação.

Rezende et al. (1998), analisando a influência do espaçamento entre aspersores na uniformidade de distribuição de água acima e abaixo da superfície do solo, constataram que os maiores valores para CUC foram obtidos no perfil do solo. Verificaram ainda, que os coeficientes obtidos acima da superfície apresentaram diferenças significativas para espaçamentos entre aspersores, enquanto abaixo da superfície, essas diferenças não diferiram significativamente. Estes autores observaram também, baseados na avaliação do desempenho do sistema, que os valores do CUC acima da superfície resultaria numa irrigação inadequada, ocorrendo o contrário, quando consideraram os valores deste coeficiente no perfil do solo. Outrossim, Penaforte et al. (1993), avaliando o desempenho de sistema de irrigação por aspersão em nove eventos de irrigação ao longo da estação de cultivo, observaram que os valores de CUC determinados na superfície do solo foram inferiores àqueles obtidos no perfil do solo.

Oliveira et al. (1993), avaliando a distribuição de água na superfície e no perfil do solo, sob irrigação por microaspersão, para distâncias radiais variando de 0 a 2,50 m, encontraram valores para CUC, na superfície do solo, variando entre 27,75 a 72,92\%, enquanto no perfil do solo os valores variaram de 85,95 a 94,72\%, devido a redistribuição da água no perfil do solo. Em estudo similar envolvendo aspersão convencional, Rocha et al. (1998), encontraram valores elevados para o CUC no perfil do solo, mesmo quando esses coeficientes na superfície foram considerados baixos. Estes autores constataram ainda, que a uniformidade no perfil do solo não foi influenciada pela uniformidade na superfície.

Constata-se então, a necessidade de determinar a uniformidade de distribuição da água no perfil do solo, a fim de verificar se a lâmina de água precipitada pelo aspersor ou microaspersor, está se redistribuindo satisfatoriamente na zona radicular da cultura. Por isto, o objetivo desta pesquisa foi avaliar a uniformidade de distribuição de água tanto na superfície como no perfil do solo para o microaspersor RainBird QN-14. 


\section{MATERIAL E MÉTODOS}

Para a determinação do Coeficiente de Uniformidade de Christiansen (Christiansen, 1972), das lâminas de água aplicadas na superfície do solo e das suas respectivas redistribuições no perfil do solo, selecionou-se uma área plana do perímetro irrigado de Bebedouro, Petrolina, num solo da classe Latossolo Vermelho Amarelo unidade 37AB, não cultivado, que foi subdividida em quadrículas de 0,50 x 0,50 m. Após a demarcação da área, foram coletadas amostras de solo nas profundidades de $0-0,20$, 0,20 - 0,40 e 0,40 - 0,60 m, em todas as quadrículas situadas no alcance do microaspersor Rain-Bird QN-14, antes da primeira irrigação, para determinação da umidade atual pelo método gravimétrico. Com base na lâmina de irrigação requerida (34,63 mm) e na intensidade média de precipitação do microaspersor $\left(1,64 \mathrm{~mm} \mathrm{~h}^{-1}\right)$, obtida em condições de laboratório, determinou-se o tempo de irrigação $(7: 00 \mathrm{~h})$, que foi dividido em três irrigações eqüivalendo aproximadamente a $1 / 3$ do tempo total. Vinte e quatro horas após a irrigação, fez-se nova coleta de amostras de solo, nas mesmas profundidades, em outros pontos distintos das quadrículas e, em seguida, outra irrigação, repetindose este procedimento até que a terceira irrigação fosse realizada. Os valores de CUC, relativos à redistribuição da lâmina de água aplicada no perfil, foram analisados através do software Statistical Analysis System (SAS). Foi utilizado o delineamento com dois fatores completamente casualizados (Irrigação e Profundidade) sem repetição, cuja interação dos fatores foi confundida com a variância residual (Anderson \& McLean, 1974).

\section{RESULTADOS E DISCUSSÃO}

Constatou-se que o valor de CUC na superfície do solo foi de $42,61 \%$, enquanto nas profundidades de 0 - 0,20, 0,20 - 0,40 e $0,40-0,60 \mathrm{~m}$ foi de $64,90,79,04$ e $84,06 \%$, respectivamente. De acordo com os resultados dos coeficientes de uniformidade no perfil, pode-se observar na Figura 1, que não houve diferença acentuada com o incremento do número das irrigações, demonstrando que a baixa uniformidade das precipitações na superfície não exerceu influência na uniformidade de distribuição da umidade no perfil do solo, ao passo que foi observada diferença com relação à profundidade das camadas (Figuras $1 \mathrm{e}$ 2). Estes resultados condizem com as afirmações de Paiva (1980) e Rocha et al. (1998) de que a uniformidade de distribuição de umidade no perfil do solo pouco depende da uniformidade da água aplicada na sua superfície. Como não constatou-se diferença significativa para os valores de CUC tanto na superfície quanto no perfil do solo ao longo das irrigações, calcularam-se os seus respectivos valores médios (Tabela 1).

Tabela 1. Valores médios de Coeficiente de Uniformidade de Christiansen na superfície e no perfil do solo

\begin{tabular}{lc}
\hline Superfície & $42,61 \%$ \\
Perfil do Solo & \\
$\quad$ Profundidade $(0-0,20 \mathrm{~m})$ & $64,90 \%$ \\
Profundidade $(0,20-0,40 \mathrm{~m})$ & $79,04 \%$ \\
$\quad$ Profundidade $(0,40-0,60 \mathrm{~m})$ & $84,06 \%$ \\
\hline
\end{tabular}

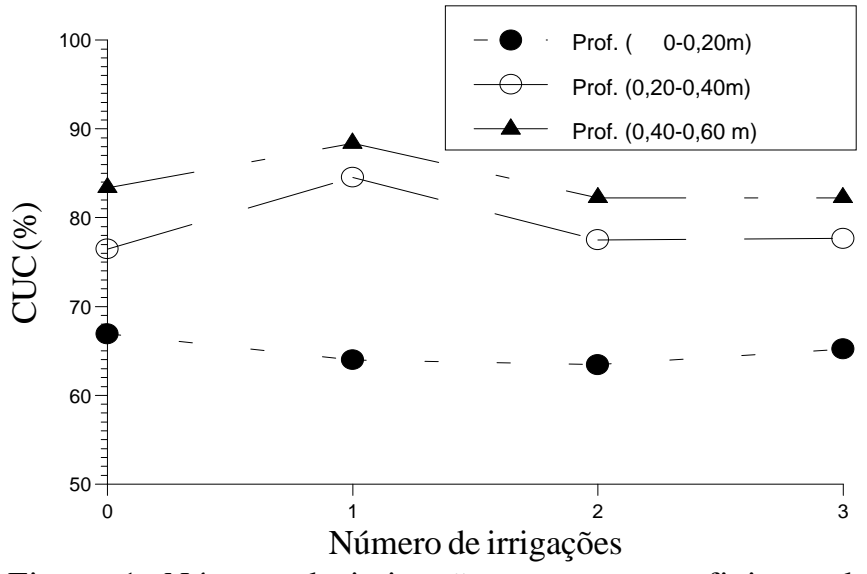

Figura 1. Número de irrigações versus coeficiente de uniformidade de Christiansen referente à distribuição de umidade para diferentes profundidades do solo

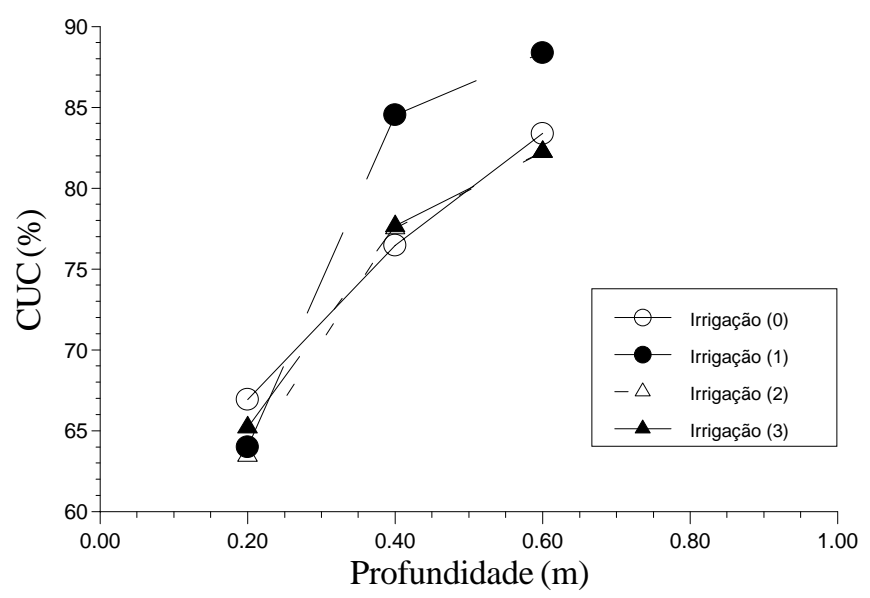

Figura 2. Profundidade versus coeficiente de uniformidade de umidade

Os valores médios de CUC mostrados na Tabela 1, obtidos na superfície e no perfil do solo, para o microaspersor RainBird QN-14, estão de acordo com Lima \& Alves (1994), que obtiveram valores de CUC inferiores a $25 \%$ na superfície do solo para microdifusores e de 27 a $62 \%$ para microaspersores, enquanto abaixo da superfície do solo os valores deste coeficiente variaram entre 40 e $50 \%$ para microdifusores e entre 52 e 57\% para microaspersores, devido à redistribuição da água no solo.

Pode-se observar através da Figura 3, que os perfis de distribuição da umidade na profundidade de $0-0,20 \mathrm{~m}$, antes das irrigações, apresentavam valores elevados de CUC, conforme mostra as grandes distâncias entre as isoietas. Pode-se observar também, um pequeno decréscimo na uniformidade de distribuição de umidade após as irrigações, demonstrando que, em função da textura desta camada, a redistribuição da água no solo sofreu influência da desuniformidade da água aplicada na superfície (Figura 3). Já nas demais profundidades, observa-se que, praticamente, não houve diferença entre a uniformidade antes e após as irrigações, demonstrando que a água aplicada teve boa redistribuição no perfil do solo (Figura 3). Isto é decorrente dos maiores 
PRIMEIRA IRRIGAÇÃO

Profundidade $0-0,20 \mathrm{~m}$

Profundidade $0,20-0,40 \mathrm{~m}$

Profundidade $0,40-0,60 \mathrm{~m}$

TERCEIRA IRRIGAÇÃO

Profundidade $0-0,20 \mathrm{~m}$

Profundidade 0,20 - 0,40 m

Profundidade 0,40 - 0,60 m
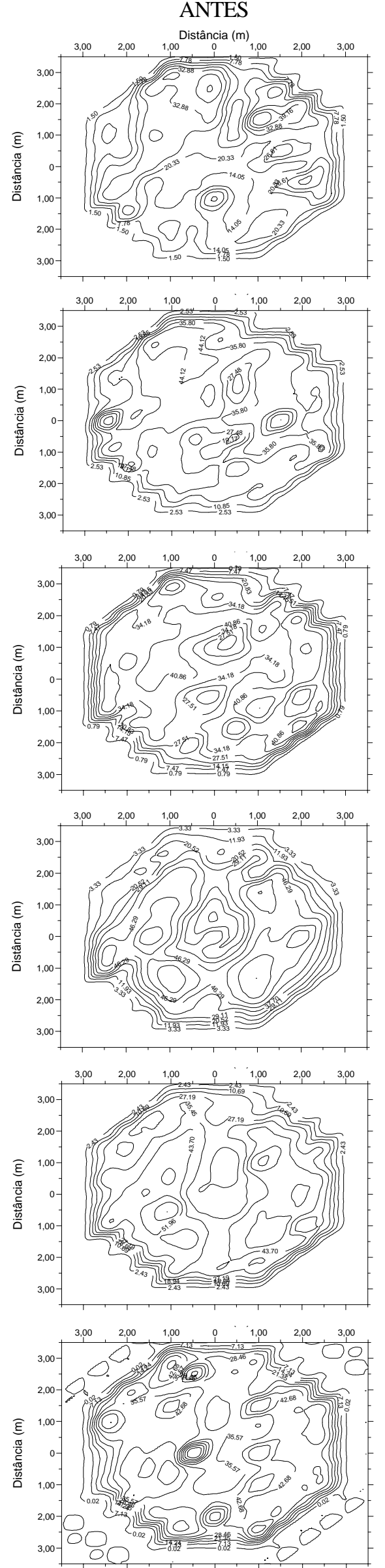
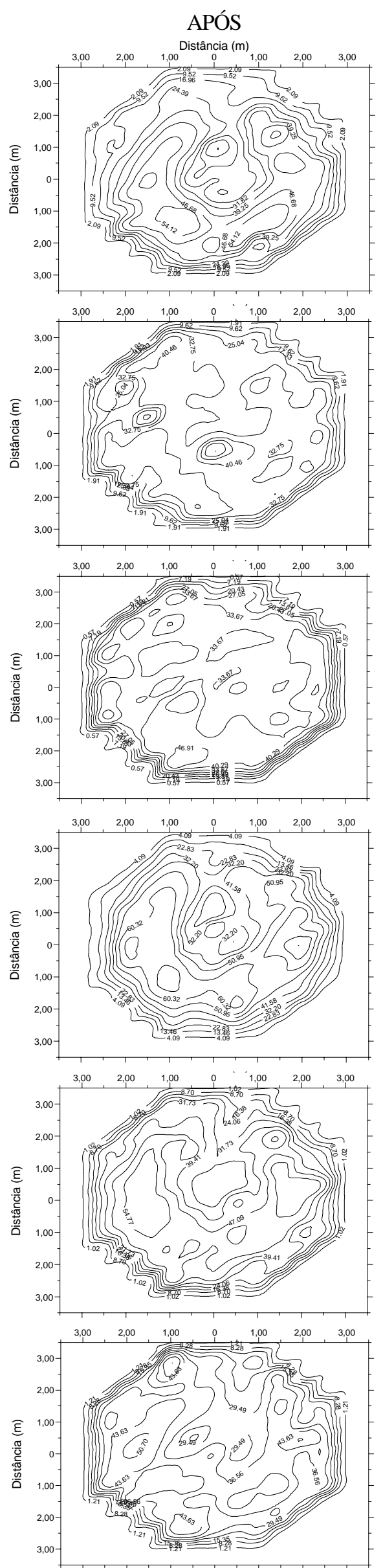

Figura 3. Isoietas da distribuição da água no perfil do solo, antes e após a primeira e terceira irrigações 
percentuais de silte e de argila existentes nas camadas de 20 - 40 e 40 - $60 \mathrm{~cm}$, relativos aos da camada de 0 - $20 \mathrm{~cm}$. Observa-se também através, das isoietas, que houve acréscimo dos valores de CUC com a profundidade.

\section{CONCLUSÕES}

1. A baixa uniformidade de precipitação do microaspersor Rain-Bird QN-14 na superfície do solo não exerceu influência na uniformidade de distribuição da umidade no perfil do solo.

2. O Coeficiente de Uniformidade de Christiansen, com relação à umidade no perfil do solo, tende a aumentar com a profundidade das camadas do solo.

3. Os valores de CUC na camada de $0-20 \mathrm{~cm}$ foi influenciada pelo valor de CUC obtido na camada superficial do solo.

\section{REFERÊNCIAS BIBLIOGRÁFICAS}

ANDERSON, V.L.; McLEAN, R.A. Some intermediate date analysis concepts. In: ANDERSON, V.L.; McLEAN, R.A. Design of experiments. New York: M. Dekker, 1974. Cap. 2, p. 40-78.

CHOUDHURY, E.N.; MILLAR, A.A. Retenção e movimento de água em Latossolo Vermelho-amarelo irrigado de Petrolina, PE. Revista Brasileira de Ciência do Solo, Campinas, v.7, n.1, p.21-26, 1983.

CHRISTIANSEN, J.E. Irrigation by sprinkling. Berkeley, California: University of California, Agricultural Experiment Station. 1972. 124 p. Reprinted Bulletin n.670, of Utah State University, Logan, 1942.

FIRAKE, N.N.; SALUNKHA, D.S. Soil moisture movement in microsprinkler irrigation. Agricultural Engineering Today. $n$. 15-16, 1992. Wallingford: CAB Abstracts 1993-7/95. CD-ROM. GOLDHAMER, D.A.; KJELGREN, R.; MOORE, L.M.; LANE, J. Low volume sprinkler surface and subsurface distribution uniformity. In: INTERNATIONAL DRIP/TRICKLE IRRIGATION CONGRESS, 3., 1985, Fresno, California. Proceedings... St. Joseph: ASAE, 1985. p. 851-858.

LIMA, L.A.; ALVES, D.R.B. Avaliação da uniformidade de distribuição de água de microaspersores e microdifusores In: CONGRESSOBRASILEIRODEENGENHARIA AGRÍCOLA, 23, 1994, Campinas. Resumos... Campinas: SBEA/UNICAMP, 1994. p.16.

LUCENA, K.F.M. de. Performance hidráulica de microgotejadores katif novos e usados. Campina Grande: UFPB, 1993. 123p. Dissertação Mestrado

MEDEIROS, J.D. Distribuição de água do microaspersor ED2 em solo com barreira impermeável no perfil. Campina Grande: UFPB, 1987. 103p. Dissertação Mestrado
OLIVEIRA, L.F.C.; SILVA, A.M.; FARIA, M.A.; LIMA, L.A. Avaliação da distribuição de água na superfície e no perfil do solo de um sistema de irrigação por microaspersão. In: CONGRESSOBRASILEIRODEENGENHARIA AGRÍCOLA, 22, 1993, Ilhéus. Anais... Ilhéus: SBEA, 1993. p.2640-2653.

PAIVA, J.B.D. de. Uniformidade de aplicação de água, abaixo da superfície do solo, utilizando irrigação por aspersão. São Carlos: USP- Escola de Engenharia de São Carlos, Departamento de Hidráulica e Saneamento, 1980. 333p. Dissertação Mestrado

PENAFORTE, A.B.; SILVA, A.M.; FARIA, M.A.; LIMA, L.A.; OLIVEIRA, M.S. Análise da performance de um sistema de irrigação por aspersão convencional, acima e abaixo da superfície do solo. In: CONGRESSO BRASILEIRO DE ENGENHARIA AGRÍCOLA, 22, 1993, Ilhéus. Anais... Ilhéus: SBEA, 1993. p.2626-2639.

PEREIRA, A.R.; FERRAZ, E.S.B.; REICHARDT, K.; LIBARDI, P.L. Estimativa da evapotranspiração e da drenagem profunda em cafezais cultivados em solos podzolizados lins e marília. Piracicaba: USP-CENA, 1974. 13p.

RAMOS, M.M.; SALES, E.S.; COUTO, L.; BERNARDO, S. Efeito da vazão e do volume de água aplicado por um emissor pontual em um Latossolo Vermelho-escuro álico, nas dimensões do bulbo molhado. Engenharia na Agricultura. Série Irrigação e Drenagem, Viçosa, v.1, n.7, p.1-16. 1993.

REICHARDT, K. Processo de transferência no sistema soloplanta-atmosfera. 4. ed. Campinas: Fundação Cargill, 1985. $445 \mathrm{p}$.

REZENDE, R.; FRIZZONE, J.A.; GONÇALVES, A.C.A.; FREITAS, P.S.L. Influência do espaçamento entre aspersores na uniformidade de distribuição de água acima e abaixo da superfície do solo. In: CONGRESSO BRASILEIRO DE ENGENHARIA AGRÍCOLA, 27, 1998, Poços de Caldas. Anais... Poços de Caldas: SBEA, 1998. p.130-132.

ROCHA, E.M.M.; NAPURUNGA, S.M.S.; COSTA, R.N.T.; CASTRO, P.T.; AGUIAR, O.S. Uniformidade e eficiência de aplicação de água da irrigação por aspersão convencional na superfície e perfil do solo In: CONGRESSO BRASILEIRODE ENGENHARIA AGRÍCOLA, 27, 1998, Poços de Caldas. Anais... Poços de Caldas: SBEA, 1998. p.286-288.

ROCHA, F.A.; LIMA, L.A. Características hidráulicas do microaspersor Antelco modelo RRN30. In: CONGRESSO BRASILEIRODEENGENHARIA AGRÍCOLA, 25, 1996, Bauru. Anais... Bauru: SBEA, 1996. p.229.

SILVA, F.F. Efeito da matéria orgânica na cultura do melão (Cucumis melo L.) sob irrigação não convencional, por potes de barro, na região semi-árida paraibana. In: CONGRESSO NACIONAL DE IRRIGAÇÃO E DRENAGEM, 8, 1988, Florianópolis. Anais... Florianópolis: ABID, 1988. v.1, p.89-105. SOUZA, V.F.; BASTOS, E.A.; SILVA, A.A.G.; AGUIAR NETTO, A.O. Uniformidade de precipitação de microaspersores sob condição de campo. In: CONGRESSO BRASILEIRO DE ENGENHARIA AGRÍCOLA, 22, 1993, Ilhéus. Anais... Ilhéus: SBEA, 1993. p.2493-2503. 